\title{
False Negative Echocardiography in an Adolescent with Anomalous Left Main Coronary Artery Origin Presenting with Cardiac Arrest
}

\author{
Kirstine N. Hansen, BMSc ${ }^{1 *}$, Jeanette K. Petersen, MD², Knud Nørregaard Hansen, MD ${ }^{1}$, \\ Lisette O. Jensen, MD, PhD, DMSci ${ }^{1}$ \\ 'Department of Cardiology, Odense University Hospital, Odense, Denmark \\ ${ }^{2}$ Department of Pathology, Odense University Hospital, Odense, Denmark
}

\begin{abstract}
A 13-year-old boy with attention deficit hyperactivity disorder treated with atomoxetin experienced cardiac collapse after physical exercise with exposure to cold water. After resuscitation using a mechanical chest compression device and treatment with extracorporeal heart lung assist, coronary angiography showed abnormal origin of the left main coronary artery and severe stenosis, which was stented. In early childhood, transthoracic echocardiography was interpreted as showing normal origins and courses of coronary arteries. The patient died, and autopsy confirmed the abnormal origin of the left main coronary artery coursing between the pulmonary artery and aorta. This case demonstrates that two-dimensional transthoracic echocardiography can lead to false negative diagnosis when assessing the origin of the left coronary artery. Thus, echocardiography may be inferior to computed tomography or magnetic resonance imaging in assessing coronary artery abnormalities.

Copyright @ 2017 Science International Corp.
\end{abstract}

\section{Key Words}

\section{Anomalous coronary artery • Extracorporeal heart lung assist • Heart attack • Echocardiogram}

\section{Introduction}

Anomalous origin of the left main coronary artery from the right sinus or anomalous origin of the right coronary artery from the left sinus are associated with ischemia and sudden death. These abnormalities are often asymptomatic and are mostly diagnosed post-mortem. Although echocardiography can be used to diagnose coronary abnormalities, its interpretation can result in false negatives [1]. These two abnormalities have a combined incidence ranging from $0.2 \%$ in autopsy studies to $1.2 \%$ in coronary angiography studies [2]. A left coronary artery running between the aorta and pulmonary artery has been linked to fatal arrhythmias and sudden death, especially in young people and usually during or after exercise [3].

\section{Case Presentation}

A 13-year-old boy experienced severe chest pain while swimming. He had a known history of attention deficit hyperactivity disorder treated with atomoxetin for several years but no history of chest pain. When paramedics arrived, the patient was awake with severe chest pain. Electrocardiography showed extensive ST segment elevations in the anterior leads and left bundle branch block. The patient went into cardiac arrest with pulseless electrical activity, and cardiopulmonary resuscitation therapy was initiated with a LUCAS mechanical chest compression device. The patient was transferred by helicopter to the nearest heart center with 24/7 invasive cardiology facilities. During transportation, the patient received adrenalin several times. After $90 \mathrm{~min}$, he arrived at the catheterization laboratory unconscious and intubated. Blood samples revealed severe

\footnotetext{
* Corresponding Author:

Kirstine N. Hansen, BMSc

Department of Cardiology

Odense University Hospital

Sdr. Boulevard 29, 5000 Odense C, Denmark

Tel.: +45 6177 2891; Fax: +45 6312 1730; E-Mail: kihan12@student.sdu.dk
} 
metabolic acidosis ( $\mathrm{pH}=6.7$ and lactate $=17 \mathrm{mmol} / \mathrm{L})$. Echocardiography showed akinetic left ventricle and reduced contraction of the right ventricle. Due to severe left-sided cardiogenic shock, extracorporeal heart lung assist with catheters through the left femoral artery and vein was initiated. Coronary angiography revealed severe stenosis of the left main stem and anomalous origin of the left coronary artery rising from the opposite sinus and running between the pulmonary artery and aorta. The right coronary artery had a normal course. The lesion in the left main coronary artery was treated with a 3.0/18-mm drug eluting stent (Figure 1), and TIMI 3 flow was achieved. Although left ventricle function was slightly improved, left ventricle ejection fraction was still severely reduced.

In the intensive care unit, the patient was treated with milrinone, norepinephrine, and epinephrine in the following hours, but he died due to cardiogenic shock with multiple organ failure and irreversible ischemic damage to his legs.

Autopsy showed an anomalous left coronary artery originating from the opposite sinus of Valsalva close to the semilunar valve, and a failed attempt to probe the lumen due to increased resistance revealed a slitlike orifice narrowing the coronary ostium. The left coronary artery had a short intramural course inside the aorta vessel wall and continued in an inter-arterial course between the aorta and pulmonary artery (Figure 2). There was ischemic damage to both ventricles, likely associated with the coronary abnormality. The heart was of normal size and weight, and the right coronary vessel showed a normal course. There was no evidence of arteriosclerosis, thrombosis, or stenosis in the coronary arteries in the non-stented areas.

Examination of the patient's pediatric medical records showed that echocardiography (Figure 3) was performed at 4 years of age on the suspicion of coronary abnormalities described in the patient's adoption information papers, which did not clarify which diagnostic tools were initially used to investigate the suspected coronary abnormalities. The echocardiography was performed by a pediatric cardiologist, and its results were described as normal with no coronary abnormalities. The indication for the echocardiography was unknown, and the patient had no history of chest pain or symptoms suggesting episodic coronary ischemia in early or late childhood.

\section{Discussion}

Anomalous coronary origin is a rare condition that can result in sudden cardiac death, most frequently when the coronary artery courses between the aorta and pulmonary artery. In active young adults with anomalous coronary origin, sudden cardiac death can be caused by exercise-induced ischemia [3]. The exact pathophysiological mechanism is not fully understood but could be related to the compression, torsion, or kinking of the coronary artery when it courses between the great vessels, particularly when blood pressure and heart rate increase [2]. Coronary artery spasms have also been mentioned as having an important role in the pathogenesis of myocardial infarction [4].

Attention deficit hyperactivity disorder is a common behavioral disorder in children and adolescents. The treatment consists of both stimulant drugs such as methyphenidate and amphetamine and non-stimulant drugs such as atomoxetine. Recently, however, the safety of these medications has come into question [5]. Atomoxetine elevates diastolic and systolic blood pressure and increases heart rate but does not significantly increase incidents of myocardial infarction and sudden death in normal people [6].

In the present case, both angiography and autopsy revealed that the left coronary artery originated from the opposite sinus of Valsalva with an intramural and inter-arterial course between the aorta and pulmonary artery. Because there were no macroscopic traces of compression of the artery or thrombosis in our case, it was not possible to determine the exact cause of the sudden obstruction of the artery, but the anomalous origin of the left coronary artery with a short intramural and subsequent inter-arterial course combined with coronary spasm may have played a significant role [4]. Thus, the patient's abnormal coronary artery anatomy, atomoxetine treatment, and coronary artery spasm provoked by cold water could together have caused the heart attack.

We re-evaluated the echocardiogram obtained when the patient was 4 years old. In the parasternal short-axis view, the left main coronary artery became visible close to the aorta at 3 o'clock after its course between the pulmonary artery and aorta and then split into left anterior descendent and left circumflex 



Figure 1. Panel A. Coronary angiography with nonselective catheterization revealed anomalous origin of the left coronary artery with thrombus in the left main stem (LM); Panel B. Contrast injection in the right coronary artery (RCA) with retrograde filling in the left coronary artery (LAD); Panel C. Balloon dilatation of the thrombotic lesion in the LM; Panel $D$. and final result after stent implantation. Left circumflex $=L C X$.

branches. This finding may have been misinterpreted as a left coronary artery with normal origin.

Two-dimensional transthoracic echocardiography coupled with Doppler color flow mapping has been the mainstay for making initial diagnosis of coronary abnormalities in children [7-9]. However, false negative diagnoses are possible owing to a failure to visualize the origin of the left coronary artery. Today, computed 


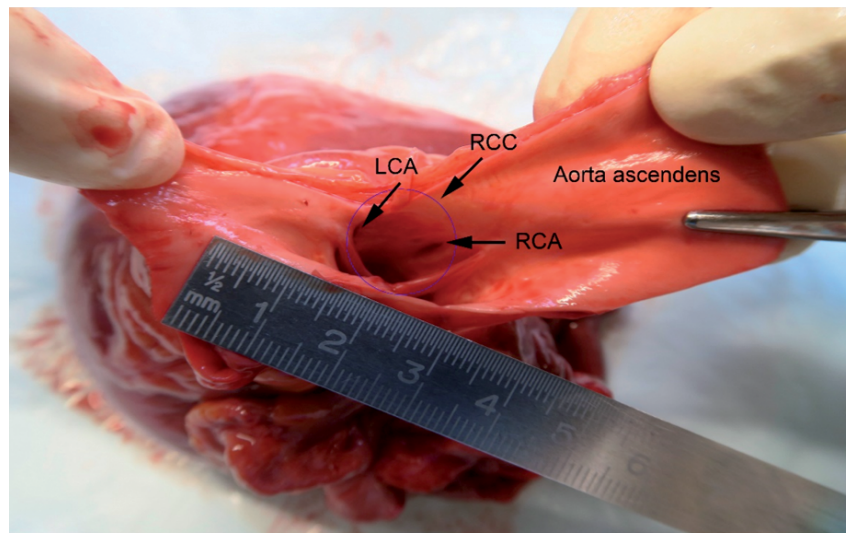

Figure 2. Autopsy findings of the heart showing the left coronary artery (LCA) with abnormal origin from the right coronary sinus. $\mathrm{RCA}=$ right coronary artery; $\mathrm{RCC}=$ right coronary cusp. tomography angiography and magnetic resonance imaging play major roles in diagnosing coronary anomalies. In case of clinical suspicion, computed tomography angiography or magnetic resonance imaging should be used to identify the origin and proximal course of coronary arteries and to evaluate cardiac function [1].

\section{Conflict of Interest}

The authors have no conflict of interest relevant to this publication.

\section{Comment on this Article or Ask a Question}



Figure 3. Parasternal short-axis echocardiogram obtained when the patient was 4 years of age. The arrow shows the left main coronary artery (LM) after its abnormal course between the pulmonary artery and aorta (Ao) splitting into the left anterior descendent and left circumflex branches. $\mathrm{RV}=$ right ventricle; $\mathrm{RA}=$ right atrium. 


\section{References}

1. Cohen MS, Herlong RJ, Silverman NH. Echocardiographic imaging of anomalous origin of the coronary arteries. Cardiol Young. 2010;20 Suppl 3:26-34. DOI: 10.1017/S104795111000106X

2. Davis JA, Cecchin F, Jones TK, Portman MA. Major coronary artery anomalies in a pediatric population: incidence and clinical importance. J Am Coll Cardiol. 2001;37:593-597. PMID: 11216984

3. Eckart RE, Jones SOT, Shry EA, Garrett PD, Scoville SL. Sudden death associated with anomalous coronary origin and obstructive coronary disease in the young. Cardiol Rev. 2006;14:161163. DOI: 10.1097/01.crd.0000197965. 99826.b2

4. Stern S, Bayes de Luna A. Coronary artery spasm: a 2009 update. Circulation. 2009;119:2531-2534. DOI: 10.1161/CIRCULATIONAHA.108.843474
5. Cooper WO, Habel LA, Sox CM, Chan KA, Arbogast PG, Cheetham TC, et al. ADHD drugs and serious cardiovascular events in children and young adults. N Engl J Med. 2011;365:1896-1904. DOI: 10.1056/NEJMoa1110212

6. Awudu GA, Besag FM. Cardiovascular effects of methylphenidate, amphetamines and atomoxetine in the treatment of attention-deficit hyperactivity disorder: an update. Drug Saf. 2014;37:661-676. DOI: 10.1007/s40264-014-0201-8

7. Taylor AJ, Rogan KM, Virmani R. Sudden cardiac death associated with isolated congenital coronary artery anomalies. J Am Coll Cardiol. 1992;20:640-647. PMID: 1512344

8. Cheitlin MD, De Castro CM, McAllister HA. Sudden death as a complication of anomalous left coronary origin from the anterior sinus of Valsalva, a not-so-minor congenital anomaly. Circulation. 1974; 50:780-787. PMID: 4419670

9. Barth CW, 3rd, Roberts WC. Left main coronary artery originating from the right sinus of Valsalva and coursing between the aorta and pulmonary trunk. J Am Coll Cardiol. 1986;7:366-373. PMID: 3944356

Cite this article as: Hanse $\mathrm{KN}$, Petersen JK, Hansen KN. False Negative Echocardiography in an Adolescent with Anomalous Left Main Coronary Artery Origin Presenting with Cardiac Arrest. Structural Heart Disease 2017;3(5):152-156. DOI: https://doi. org/10.12945/j.jshd.2017.021.17 